felt additional training would be valuable. These included greeting and interacting with relatives, communication skills, cultural competence and the symptoms and signs of advanced illness. There was some disagreement about how training contributed to professionalisation and the extent of what education should be provided with respect to role boundaries. Volunteers advocated a mixed-methods approach to training, with an emphasis on scenario-based teaching and on-the-job mentoring and support.

Conclusion This study suggests areas of training that inpatient hospice volunteers perceive as worthwhile. It will inform local practice, shaping the education and support of future volunteers, and add to the wider evidence-base surrounding the development of this crucial group within the hospice workforce.

\section{0-15 A SHARED CARE PATHWAY FOR PEOPLE WITH ADVANCED LIVER DISEASE (ALD); INNOVATION OR IDEALISTIC?}

Virginia Campbell, Sharon Quinn. St Luke's Hospice, Basildon, UK

\subsection{6/bmjspcare-2017-hospice.15}

Background People with ALD have complex end-of-life needs. Palliative care provision or clear national guidance is limited. An innovative, unique joint hepatology/specialist palliative care project exploring a parallel planning approach has been established between the hospice and secondary care, which will lead the learning for hospices around ALD and non-malignant conditions.

Aims To explore the impact of this shared care approach on patient and carer outcomes and experience.

Methods Patients were recruited by the hospice Specialist Nurse Practitioner (SNP) from hepatology hospital clinics. The SNP offered holistic needs assessment, opportunities to discuss advance care planning and access to supportive care at the hospice whilst patients remained under hepatology management. Patients were reassessed four to eight weekly using OACC measures and the Short Form Liver Disease Quality of Life Assessment. Carers were assessed using CSNAT. In addition to analysis of patient data, hospital admission, A and E and GP visit avoidance was evaluated to assess health economics and quality of life.

Results A total of 38 patients were registered. At baseline our sample $(n=36)$ had a mean I-POS of 27.4 (range 4-64) and a mean AKPS score of 68.3(range 50.90). At the four month assessment, the sample $(n=23)$ had a mean I-POS of 19.7 (range 1-42) and a mean AKPS score of 77.0 (range 50-90). Preliminary analysis suggests that patients receiving early hospice intervention have reduced symptom burden and improved performance status. This was further supported by focus group feedback which highlighted continuity of care and open communication as contributing to improved well-being.

Conclusions Final data will be presented at the conference demonstrating key learning points from this collaborative and innovative approach which will lead and inform future service provision across hospices. Early and timely introduction to hospice services is beneficial to this marginalised group and this model is replicable for people with other non-malignant conditions.

\section{0-16 IMPROVING END OF LIFE CARE FOR ADULTS WITH SEVERE MENTAL ILLNESS AND LIFE-LIMITING CONDITIONS}

1,2,3 Jed Jerwood, 'Jane Coad, 'Diane Phimister, ${ }^{1}$ Nikki Holliday. ${ }^{1}$ Coventry University, Coventry, UK; ${ }^{2}$ John Taylor Hospice, Birmingham, UK; ${ }^{3}$ Birmingham and Solihull Mental Health Trust, UK

\subsection{6/bmjspcare-2017-hospice. 16}

Background People with mental illness experience higher rates of many life-limiting conditions and die on average twenty years earlier than the general population. A literature review was carried out which revealed limited understanding of the end of life needs of this patient group. The role of clinical staff was highlighted as a key factor, yet their views were not reflected in the published research.

Aims The aim of this research was to conduct an exploratory study to develop further understanding of how to improve end of life care for people with severe mental illness through exploring the views and experiences of clinical staff working in both mental health and end of life services.

Methods Four focus groups with clinicians were conducted, using the CUbe method (Magee, Fielden, \& Moody, 2015), across a large Mental Health Trust and a hospice. 23 participants from a range of professional backgrounds attended and shared their experiences and perceptions of the barriers to delivering good end of life care to people with mental illnesses. The data was analysed using the Framework Method (Gale, Heath, Cameron, Rashid, \& Redwood, 2013) and themes and explanatory concepts were drawn.

Findings The findings of the study were clustered into five themes: Structure of The System, Presentation of the Patient, Confidence of the Clinician and the Problem of Partnership. Implications for practice were drawn from the themes which can be used to inform improvements to clinical practice.

Conclusions The barriers to providing good end of life care were described within the literature, which largely originated outside the UK. The findings of the analysis found many similarities and some new findings. Recommendations were made which include improvements to partnership working, involvement of patients, carers and clinical staff in improving care and the need to develop the confidence and knowledge of clinicial staff from mental health and end of life services.

\section{Parallel sessions 5}

\section{Reaching out: primary care, care homes and ambulance services}

\section{0-17 GPs' EXPERIENCES AND PERCEPTIONS OF A HOSPICE SERVICE: A QUALITATIVE EVALUATION}

Sarah Onions, Sarah Mitchell, Jeremy Dale. University of Warwick, Warwick, UK

10.1136/bmjspcare-2017-hospice. 17

Background Most palliative and end of life care (PEOLC) is provided in primary care with referral to specialist palliative care (SPC) services for patients with more complex problems. Collaboration between general practitioners (GPs) and hospices 
is essential to deliver patient-centred PEOLC. There is little in-depth research on the collaboration between general practice and hospice services and the variety of models and processes that currently exist.

Aims To investigate GP experiences and perceptions of hospice care and the facilitators and limitations to SPC referral and shared care management.

Method A GP interview study across a CCG. Semi-structured interviews were performed and a framework methodology employed.

Results Twelve GPs participated. They described how use of hospice services is influenced by their own experience; practice expertise; perceptions of patients' needs; and relationships with the hospice. Key themes were GP factors; perceived accessibility and responsiveness of hospice services; patient and family factors, which affect referral decision-making; and effective professional relationships. GPs viewed urgent referrals or referrals for patients from ethnic minorities as most challenging. They also struggled to identify the right time to refer patients with non-malignant diagnoses. GPs uniformly preferred personal communication with clinical nurse specialists and relied on stable professional relationships suggesting that care was disrupted when CNS cover was lacking.

Conclusions This study gives weight to the challenge of partnership working across teams of different palliative care professionals, and illustrates how professional relationships are central to effective collaboration, referral and patient management. We recommend further research into robust collaborative working and an evaluation of equity of access to services particularly for ethnic minorities. We also suggest the need for further development of contemporaneous electronic patient notes and GP education. These with stable service delivery are vital to planning future models of care and proactive service delivery.

\section{0-18 GSF AND HOSPICE PARTNERSHIP WORKING AS REGIONAL CENTRES DELIVERING GSF CARE HOMES TRAINING}

Keri Thomas, Clare Fuller, Denise Douglas, Annabel Foulger, Anne Keating. Gold Standards Framework Centre C.I.C. Shrewsbury, UK

10.1136/bmjspcare-2017-hospice.18

Background Care homes, where a fifth of the population die, are crucial providers of end-of-life care (EOLC), for the elderly with frailty and dementia, but many require further EOLC training. The Gold Standards Framework Care Homes (GSFCH) Training Programme provides a more proactive, person-centred, systematic approach adopted within care homes, enabling better care with fewer avoidable admissions and hospitals deaths, and more dying where they choose. The GSF Centre developed partnerships with several hospices as Regional Centres as part of the national cascade of the GSFCH Programme.

Aims To describe the development of GSF Regional Centre to cascade training to facilitate embedded sustainable proactive end-of-life care for residents in line with their wishes and preferences.

Methods Twelve regional centres were recruited through an application process and a process of staged train-the-trainers developed ensuring quality assurance of the GSF Care Homes Programme delivery to a wider local audience, usually involving four to six workshops over several months. Training included earlier identification, clinical assessment, advance care planning discussions and planning living and dying well. Intrinsic comparative evaluation data is collated to evaluate the impact. Many homes then progress to Accreditation with the recognised GSF Quality Hallmark Award and many more are re-accredited, demonstrating sustainability.

Results Results from the Regional Centres delivery of the GSF $\mathrm{CH}$ Programme will be presented including numbers trained, attainment of standards, and impact factors. The benefits of a collaborative approach are further discussed showing the benefit to hospices of this shared approach.

Conclusion Partnership working between GSF Centre, hospice regional centres and care homes facilitates spread of this successful programme, benefits to hospices with increased uptake of other training and benefits to care homes enabling more residents receiving better EOLC and more dying where they choose. Further areas of development will be discussed.

\section{0-19 EVALUATION OF THE 'HOSPICE IN YOUR CARE HOME' PROJECT}

1,2Katherine Froggatt, 1,2Nancy Preston, 1,2Rachael Eastham, 1,2Garuth Chalfont, ${ }^{1,2}$ Debbie Dempsey. International Observatory on End of Life Care, Lancaster University, Lancaster, UK; ${ }^{2}$ Wigan and Leigh Hospice, Wigan, UK

\subsection{6/bmjspcare-2017-hospice. 19}

Background There is limited evidence on the impact of multicomponent interventions to support the delivery of palliative care in care homes. In 2015, one hospice established an innovative 'Hospice in Your Care Home' team using a number of interventions: role modelling and working alongside staff, responses to urgent referrals, advance care planning and training courses. This project has been externally evaluated.

Aims

- To evaluate the process and outcomes of the 'Hospice in Your Care Home' initiative, with specific reference to hospital admissions and end of life care practices.

- To ascertain the costs of delivering the project.

- To identify facilitators and barriers to the implementation of the initiative.

Methods The responsive evaluation comprised:

- an analysis of secondary service provision data

- focus group interviews with care home managers $(n=7)$, care home staff $(n=11)$ and the project team $(n=6)$

- preliminary analysis of cost (time and finance).

Secondary data was analysed using descriptive and inferential statistics. Framework analysis structured the qualitative data analysis.

Results Nine care homes participated with one facility leaving and one joining part way through. Hospital admissions were significantly reduced by $25 \%(\mathrm{p}=0.01)$, between 2015 and 2016. Resident status meetings were conducted in each facility, with 4479 residents discussed at 217 meetings. Staff described increased confidence in their ability to care for residents with palliative care needs. To establish this project three stages of implementation were identified: initiation, assimilation, and 'everyday' running. The project was facilitated by the hospice team's flexibility and supportive approach. Reported barriers reflected the ongoing challenges regarding staffing levels and release of staff to attend training. 